\title{
Development of Free Vortex Wake Method for Yaw Misalignment Effect on the Thrust Vector and Generated Power
}

\author{
Hamidreza Abedi* \\ Chalmers University of Technology, 412 96, Gothenburg, Sweden \\ Lars Davidson, ${ }^{\dagger}$ \\ Chalmers University of Technology, 412 96, Gothenburg, Sweden \\ Spyros Voutsinas, \\ National Technical University of Athens, Athens, Greece
}

\begin{abstract}
Wind power is currently one of the most reliable new energy sources serving as an alternative to fossil fuel generated electricity and is known as a widely distributed clean and renewable source of energy. It is now the world's fastest growing energy source and has also become one of the most rapidly expanding industries. The aerodynamics of a wind turbine is governed by the flow around the rotor, where the prediction of air loads on rotor blades in different operational conditions and their relation to rotor structural dynamics is crucial for design purposes. One of the challenges in wind turbine aerodynamics is the yaw condition where the undisturbed upstream flow is not perpendicular to the rotor plane, giving a non-uniform blade load which is contrary to the axisymmetric flow assumption in the BEM (Blade Element Momentum) method. However, there are some engineering methods modifying the BEM method for yaw misalignment situations, ${ }^{1}$ where they often calculate the skewed axial induction factor as an average value over the rotor disk which is insensitive to the blade rotation direction. On the other hand, experiments show that the thrust vector for a positive yaw misalignment differs from that for a negative yaw misalignment. A free vortex wake method, based on the potential, inviscid and irrotational flow, is developed to study the deviation of thrust vector relative to rotor shaft. The results are compared with the BEM method ${ }^{2}$ and experimental data. A two-bladed variable speed wind turbine, the Hönö wind turbine, ${ }^{3}$ is used for this study.
\end{abstract}

\section{Nomenclature}

BEM Blade Element Momentum

CFD Computational Fluid Dynamics

$\Gamma \quad$ Circulation, $\mathrm{m}^{2} / \mathrm{s}$

$\vec{V} \quad$ Velocity vector, $\mathrm{m} / \mathrm{s}$

$\Omega$ Rotational velocity, $\mathrm{rad} / \mathrm{s}$

$\vec{r} \quad$ Position vector, $\mathrm{m}$

$\gamma \quad$ Vorticity distribution, $\mathrm{m} / \mathrm{s}$

$\alpha \quad$ Angle of attack, deg

$t$ time, s

$\vec{V}_{\infty}$ free stream velocity vector, $\mathrm{m} / \mathrm{s}$

$\overrightarrow{d l} \quad$ Length vector, $\mathrm{m}$

$\rho \quad$ Air density, $\mathrm{kg} / \mathrm{m}^{3}$

$\vec{L}^{\prime} \quad$ Lift force vector per unit span length , N/m

$\vec{D}^{\prime} \quad$ Drag force vector per unit span length, $\mathrm{N} / \mathrm{m}$

${ }^{*}$ Ph.D. Candidate, Division of Fluid Dynamics, Department of Applied Mechanics

${ }^{\dagger}$ Professor, Division of Fluid Dynamics, Department of Applied Mechanics

¥Associate Professor, Fluid Section, School of Mechanical Engineering 


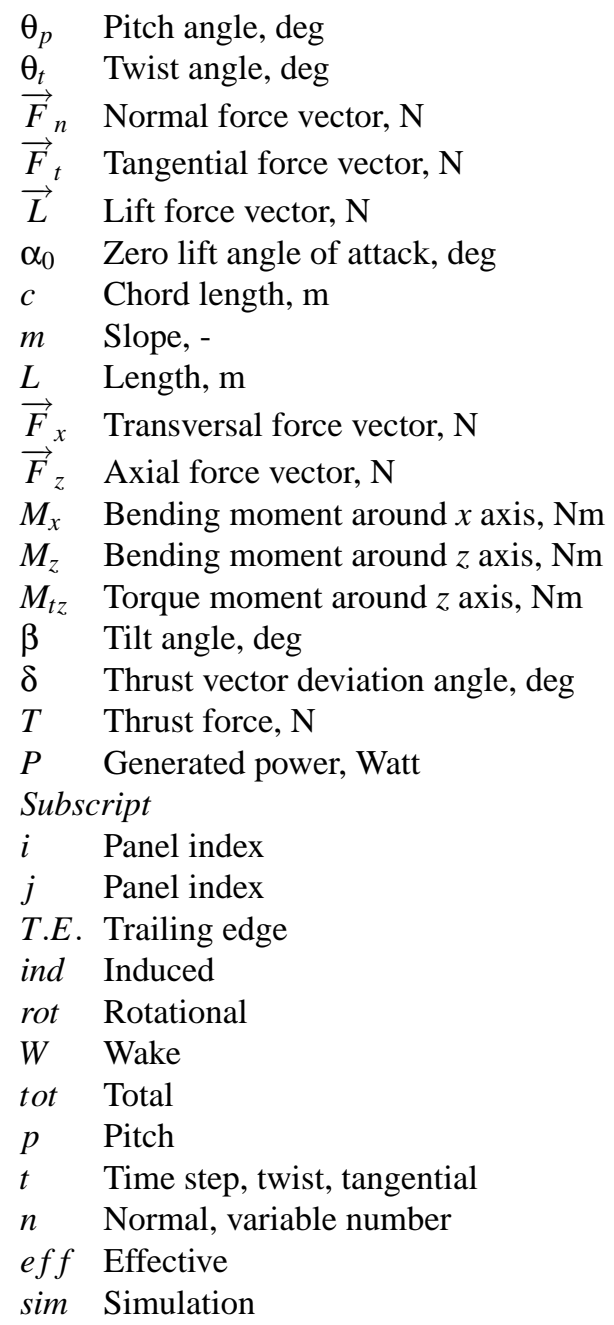

\section{Introduction}

Among clean energy sources that are renewable, wind is regarded as the least destructive to the environment. By the exponential growth of wind turbines all around the world, and its general acceptance among people, the demand and its worthwhileness makes it apt for research, especially to enhance its performance.

According to data provided by the Renewables Global Status Report in 2013, wind capacity increased globally by $19 \%$, the increase being $45 \mathrm{GW}$. That is, it reached $283 \mathrm{GW}$ to a record high despite the uncertainty in the policy in the key markets.

There are different methods for modelling the aerodynamics of a wind turbine with different levels of complexity and accuracy, such as the Blade Element Momentum (BEM) theory and solving the Navier-Stokes equations using Computational fluid Dynamics (CFD).

Today, engineering methods based on the BEM method are used extensively for analyzing the aerodynamic performance of a wind turbine. The BEM model is based on the steady and homogeneous flow assumption and that aerodynamic loads act on an actuator disc instead of a finite number of blades. The BEM method is computationally fast and is easily implemented, but it is acceptable only for a certain range of flow conditions. ${ }^{2}$ A number of empirical and semi-empirical correction factors have been added to the BEM in order to increase its application range, such as yaw misalignment, dynamic inflow, finite number of blades and blade cone angle, ${ }^{4}$ but they are not relevant to all operating conditions and are often incorrect at high tip speed ratios where wake distortion is significant. ${ }^{5}$ Among these corrections, the yawed flow modifications for the BEM method is very important. There are different engineering approaches such as disk averaged induced velocity, advancing and retreating blade effect, skewed wake geometry with trailing vortices and skewed wake geometry with root vorticity where they are originally based on the disk averaged 
velocity on an annular ring by Glauert. ${ }^{6}$

In this study, the skewed wake geometry with trailing vortices model, which is based on the oblique cylindrical vortex wake, is used ${ }^{7}$ to modify the BEM method implementation under yawed flow conditions. According to Ref. 7, 1 , the skewed wake geometry only modifies the axial induction factor and it depends on the angle between the trailing wake and the rotor axis (skew angle), the blade element radial position and the blade azimuthal angle with respect to the rotor plane. In this model, the tangential induction factor is not corrected which leads to overprediction of the tilt moment as a result of yaw condition, especially for vertical wind shear flow.

The vortex theory, which is based on the potential, inviscid and irrotational flow can be used to predict the aerodynamic performance of wind turbines. It has been widely used for aerodynamic analysis of airfoils and aircrafts. Although the standard method cannot be used to predict viscous phenomena such as drag and boundary layer separation, its combination with tabulated airfoil data makes it a powerful tool for the prediction of fluid flow. Compared with the BEM method, the vortex method is able to provide more physical solutions for attached flow conditions with boundary layer corrections, and it is also valid over a wider range of turbine operating conditions. Although it is computationally more expensive than the BEM method, it is still feasible as an engineering method.

In vortex methods, the trailing and shed vortices are modeled by either vortex particles or vortex filaments moving either freely, known as free wake ${ }^{8-10}$ or restrictedly by imposing the wake geometry known as prescribed wake. ${ }^{11,12}$ The prescribed wake requires less computational effort than the free wake, but it requires experimental data to be valid for a broad range of operating conditions. The free wake model, which is the most computationally expensive vortex method, is able to predict the wake geometry and loads more accurately than the prescribed wake because of less restrictive assumptions. Therefore, it can be used for the load calculations for different flow conditions. However, its application is limited to attached flow and it must be linked to tabulated airfoil data to predict air loads in the presence of drag and flow separation. The advantage of the free wake model to yawed flow is that it can be applied for both axisymmetric and asymmetric upstream flow conditions. Therefore, all different engineering approaches mentioned above for the BEM method are naturally taken into account.

The deviation of thrust vector relative to the generator shaft and wind direction is known as one of the vibration sources for a wind turbine in large yaw misalignment. The aim of this paper is to find the resulting thrust vector with respect to the turbine shaft and the power generation for different yaw misalignments and wind speeds. Two aerodynamic load calculation methods, the time-marching vortex lattice free wake and the BEM method are used in the present study, and they are compared with experimental data for validation.

\section{Model}

\section{II.A. Assumptions}

Each engineering model is constructed based on some assumptions. Here, some of those are discussed. In this study, the upstream flow is assumed to be constant in time (steady state) whereas its speed varies in the vertical direction called vertical wind shear following the power law formulation and its direction varies with respect to the horizontal plane called yaw condition. Blades are assumed to be rigid, so the elastic effect of the blades is neglected. Because

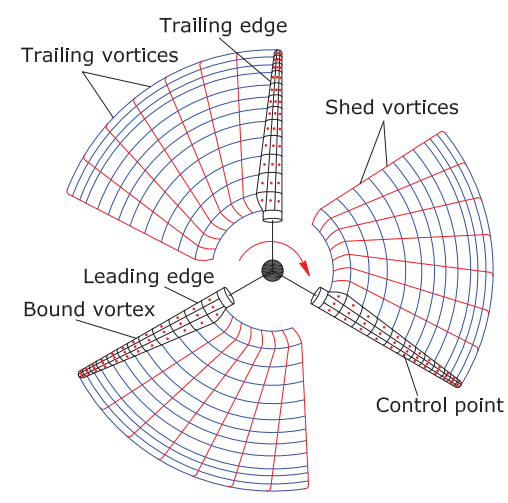

Figure 1. Schematic of vortex lattice free wake

of the large circulation gradients $(d \Gamma / d r)$ near the tip and the root of the rotor blade, the blade radial segmentation (in the spanwise direction) is refined by the cosine rule ${ }^{4}$ whereas the blade elements, in the chordwise direction, are distributed at equi-distant increments. 
In the vortex lattice free wake model, a finite number of vortex wake elements move freely based on the local velocity field, and contrary to the prescribed wake model, allowing wake expansion as well. Each vortex wake element contains two points, one at the head (A), and another at the tail (B) which are known as Lagrangian markers, where the induced velocity components are calculated using the Biot-Savart law; their movements give rise to the wake deformation. The vortex flow theory assumes that the trailing and shed wake vortices extend to infinity. However, since the effect of the induced velocity field by the far wake is small on the rotor blade, the wake in the present study extends only four diameters downstream of the wind turbine rotor plane.

\section{II.B. Vortex Lattice Free Wake (VLFW)}

The vortex lattice method is based on the thin lifting surface theory of vortex ring elements, ${ }^{13}$ where the blade surface is replaced by vortex panels that are constructed based on the airfoil camber line of each blade section (see figure 2). To take the blade surface curvature into account, the lifting surface is divided into a number of panels both in the chordwise and spanwise directions, where each panel contains the vortex ring with strength $\Gamma_{i j}$ in which $i$ and $j$ indicate panel indices in the chordwise and spanwise directions, respectively. The strength of each blade bound vortex ring element, $\Gamma_{i, j}$, is assumed to be constant and the positive circulation is defined on the basis of right-hand rotation rule. In order to fulfill the $2 \mathrm{D}$ Kutta condition (which can be expressed as $\gamma_{T . E} .=0$ in terms of the strength of the vortex sheet) the leading segment of a vortex ring is located at the $1 / 4$ panel length (see figure 3 ). The control point of each panel is located at $3 / 4$ of the panel length meaning that the control point is placed at the center of the panel's vortex ring.

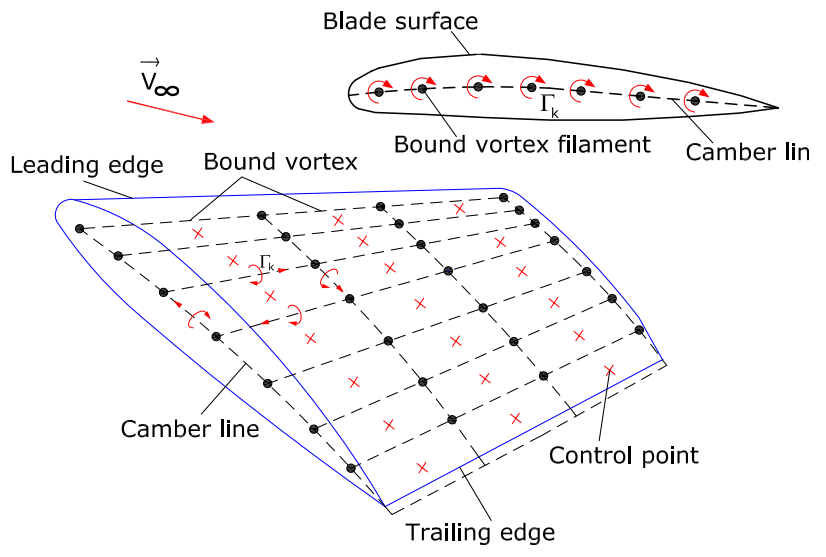

Figure 2. Lifting surface and vortex panels construction

The wake elements which induce a velocity field around the blade are modeled as vortex ring elements, and they are trailed and shed based on a time-marching method. To satisfy the 3D trailing edge condition for each spanwise section, the strength of the trailing vortex wake rings must be equal to the last vortex ring row in the chordwise direction $\left(\Gamma_{\text {T.E. }}=\Gamma_{\text {Wake }}\right)$. The flow tangency condition at each blade control point must be specified to find the blade bound vortices strength $\left(\Gamma_{i, j}\right)$ at each time step. The velocity components at each blade control point include the free stream $\left(\mathbf{V}_{\infty}\right)$, rotational $(\Omega \mathbf{r})$, blade vortex rings self-induced $\left(\mathbf{V}_{\text {ind,bound }}\right)$ and wake induced $\left(\mathbf{V}_{\text {ind,wake }}\right)$ velocities where $\mathbf{V}_{\infty}, \Omega \mathbf{r}$ and $\mathbf{V}_{\text {ind,wake }}$ are known at each time step. The blade is assumed to be rigid, hence the blade self-induced components, called influence coefficients, are constant at each time step, and they are computed only once. However, if the blade is modeled as a flexible blade, they must be calculated at each time step. At the first time step (see figure 4), there are no free wake elements. At the second time step (see figure 5), when the blade is rotating, the first wake panels are shed. Their strength is equal to the bound vortex circulation of the last row of the blade vortex ring elements (Kutta condition), located at the trailing edge at the previous time step, which means that $\Gamma_{W_{t_{2}}}=\Gamma_{T . E . t_{1}}$, where the $W$ and $T . E$. subscripts represent the wake and the trailing edge, respectively.

At the second time step, the strength of the blade bound vortex rings is calculated by specifying the flow tangency boundary condition where, in addition to the blade vortex ring elements, the contribution of the first row of the wake panels is considered. This methodology is repeated, and the vortex wake elements are trailed and shed at each time step, where their strengths remain constant (Kelvin theorem) and their corner points are moved based on the governing equation (Eq.(1) ) by the local velocity field, including the wind velocity and the induced velocity by all blade and wake vortex rings (see figures 4 and 5). The governing equation for the wake geometry is 


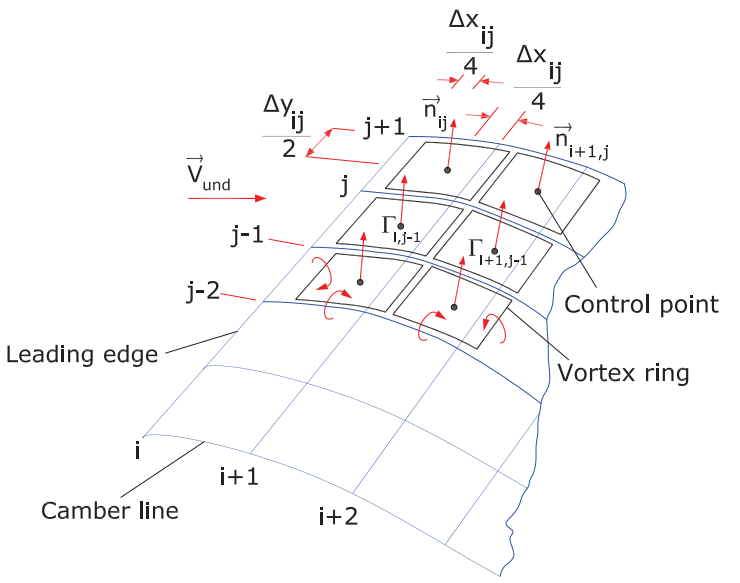

Figure 3. Numbering procedure

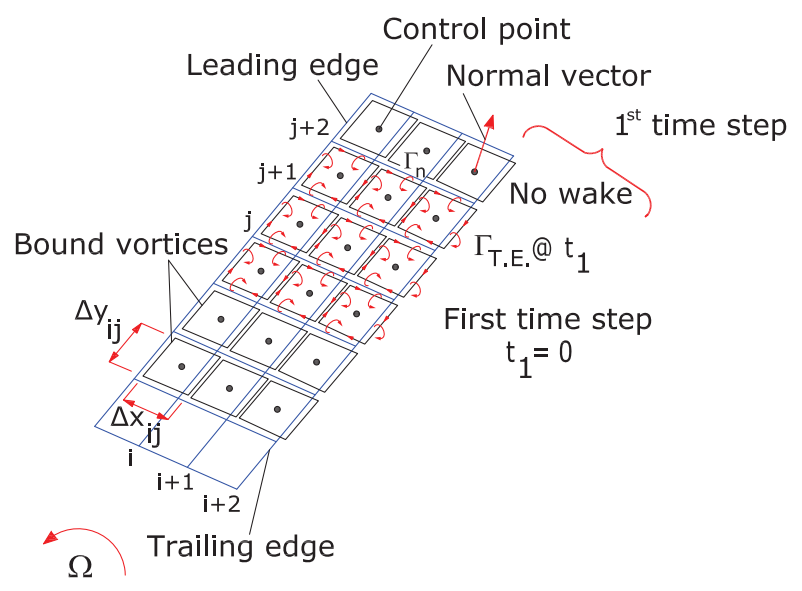

Figure 4. Schematic of wake evolution at the first time step

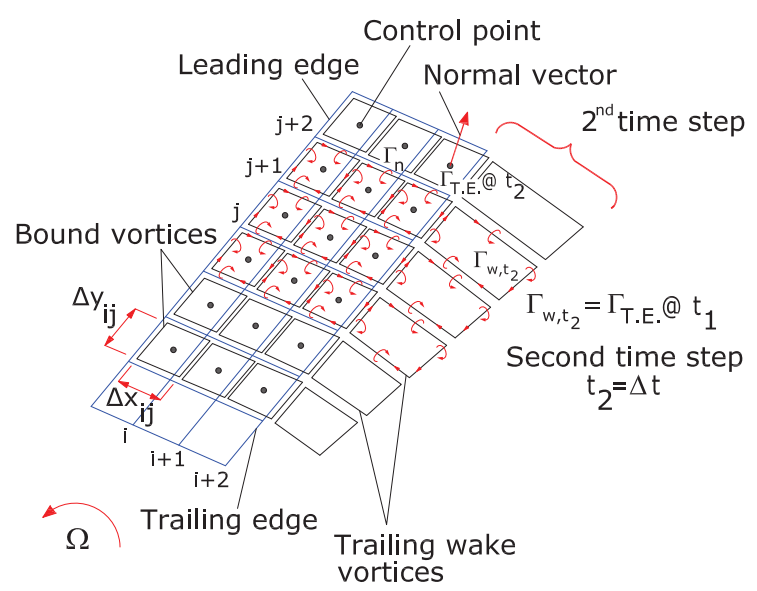

Figure 5. Schematic of wake evolution at the second time step 


$$
\frac{d \vec{r}}{d t}=\vec{V}(\vec{r}, t) \quad \vec{r}(t=0)=\overrightarrow{r_{0}}
$$

where $\vec{r}, \vec{V}$ and $t$ denote the position vector of a Lagrangian marker, the total velocity field and time. The total velocity field, expressed in the rotating reference frame i.e. $\vec{V}_{r o t}=0$, can be written as

$$
\vec{V}_{\text {tot }}=\vec{V}_{\infty}+\vec{V}_{\text {ind,blade }}+\vec{V}_{\text {ind,wake }}
$$

Different numerical schemes may be used for Eq.(1) such as the explicit Euler method, the implicit method, the Adams-Bashforth method and the Predictor-Corrector method. The numerical integration scheme must be considered in terms of the accuracy, stability and computational efficiency. Here, the first-order Euler explicit method is used as

$$
\vec{r}_{t+1}=\vec{r}_{t}+\vec{V}_{t o t}\left(\vec{r}_{t}\right) \Delta t
$$

where $\vec{V}$ is taken at the old time step.

\section{Load Calculation}

In the vortex flow, the only force acting on the rotor blades is the lift force which can be calculated either by the Kutta-Jukowski theory or the Bernoulli equation where the viscous effects such as the skin friction and the flow separation are not included. Therefore, in order to take into account viscous effects and flow separation, it must be combined with the aerodynamic coefficients through the tabulated airfoil data.

Two models are used in the present work, the standard potential method and the 2D static airfoil data method which are based on the quasi-static assumption.

Our standard potential method is based on the thin lifting surface theory of vortex ring elements, where the body is part of the flow domain. Therefore, the effective angle of attack is calculated based on the dynamic approach (force field) by projecting the lift force acting on the rotor blades into the normal and tangential directions with respect to the rotor plane.

In the standard potential method, the airfoil characteristic of each spanwise section is not taken into account. In addition, the predicted angle of attack, computed on the basis of the potential flow solution (i.e. the lifting surface theory), is always greater than that calculated by the viscous flow, which means that it cannot be directly used as entry to look up the tabulated airfoil data to provide the aerodynamic coefficients.

To overcome these restrictions, the 2D static airfoil data method is proposed. In the 2D static airfoil data method, the new angle of attack is calculated by using the tabulated airfoil data where it is directly connected to both tabulated airfoil data and the potential solution parameter $(\Gamma)$. This angle of attack is used as the entry to look-up the airfoil table and then we are able to calculate the aerodynamic coefficients giving the lift and drag forces for each blade element.

\section{III.A. The Standard Potential Method}

In the VLFW method, when the position of all the Lagrangian markers is calculated in each time step, we are able to compute the velocity field around the rotor blade where, as a consequence, the lift force can be calculated according to the Kutta-Jukowski theorem which in differential form reads as

$$
\overrightarrow{d L}=\rho \vec{V} \times \Gamma \overrightarrow{d l}
$$

where $\rho, \vec{V}, \Gamma$ and $\overrightarrow{d l}$ denote air density, velocity vector, vortex filament strength and length vector, respectively. The Kutta-Jukowski theorem is applied at the mid-point of the front edge of each blade vortex ring and gives the potential lift force where the lift force of each spanwise blade section is calculated by summing up the lift force of all panels along the chord. The lift force for each blade panel except the first row near the leading edge is computed by

$$
\vec{L}_{i, j}=\rho \vec{V}_{t o t, i, j} \times\left(\Gamma_{i, j}-\Gamma_{i-1, j}\right) \Delta \vec{y}_{i, j}
$$

For the blade panels adjacent to the leading edge, Eq.(5) can be written as

$$
\vec{L}_{1, j}=\rho \vec{V}_{t o t, 1, j} \times \Gamma_{1, j} \Delta \vec{y}_{1, j}
$$




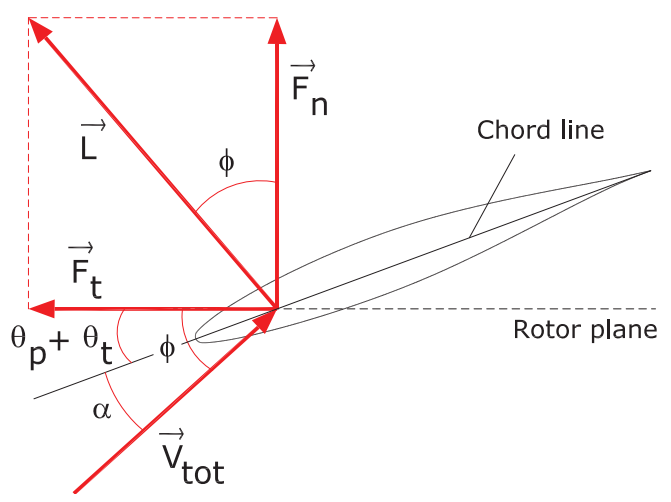

Figure 6. Potential load decomposition

where $\vec{V}_{t o t, i, j}$ is computed as Eq.(2). The total lift of each blade section in the spanwise direction is obtained as

$$
\vec{L}_{j}=\sum_{i=1}^{N} \vec{L}_{i, j}
$$

where $N$ denotes the number of chordwise sections. Decomposition of the lift force for each blade spanwise section into the normal and tangential directions with respect to the rotor plane (see figure 6) gives the effective potential angle of attack for each section.

$$
\alpha=\tan ^{-1}\left(F_{t} / F_{n}\right)-\theta_{t}-\theta_{p}
$$

where $\alpha, F_{t}, F_{n}, \theta_{t}$ and $\theta_{p}$ represent the effective angle of attack, tangential force, normal force, blade section twist and blade pitch, respectively.

\section{III.B. 2D Static Airfoil Data Method}

For the thick airfoil, commonly used in wind turbine blades, the thin airfoil theory which is expressed by the linear relation of the lift coefficient and the angle of attack, is no longer valid. Consequently the higher the lift the higher the angle of attack; thus, considerable lift reduction due to flow separation at higher angles of attack cannot be predicted.

As described in the load calculation section, the 2D static airfoil data method is introduced to increase the applicability of the VLFW method. This can be done by modification of the angle of attack obtained from the standard potential method together with the tabulated airfoil data.

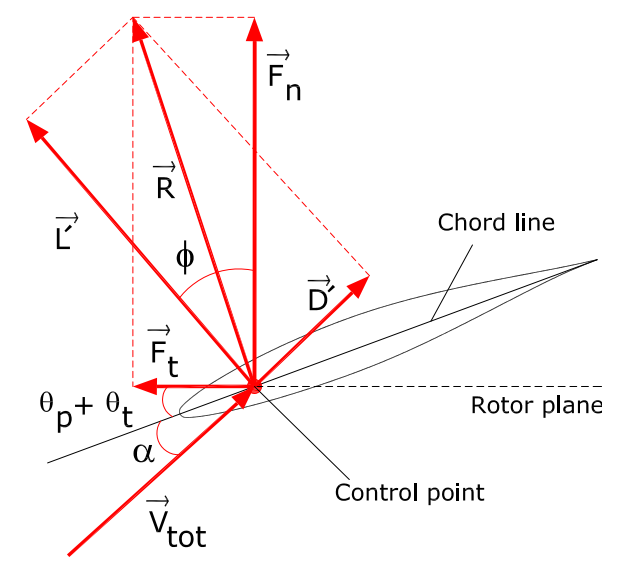

Figure 7. Viscous load decomposition

According to the Kutta-Jukowski theory, the magnitude of the lift force per unit spanwise length, $L^{\prime}$, is proportional to the circulation, $\Gamma$, and it is given by

$$
L^{\prime}=\rho V_{t o t} \Gamma
$$


where $\rho, V_{t o t}$ denote the air density and the total velocity magnitude, respectively. The circulation for each spanwise section is equal to the bound vortex circulation of the last row vortex ring element, located at the trailing edge. In addition, in the potential flow, the lift coefficient, expressed by the thin airfoil theory, is a linear function of angle of attack (with constant slope equal to $2 \pi$ ) and it is given by

$$
C_{L}=m\left(\alpha-\alpha_{0}\right)
$$

where $m=2 \pi, \alpha$ and $\alpha_{0}$ indicate the slope, the angle of attack and the zero-lift angle of attack, respectively.

The lift coefficient is generally defined as

$$
C_{L}=\frac{L^{\prime}}{0.5 \rho V_{t o t}^{2} c}
$$

where $c$ denotes the airfoil chord length. Combination of Eqs.(9), (10) and (11) gives the modified angle of attack as

$$
\alpha=\frac{2 \Gamma}{m V_{t o t} c}+\alpha_{0}
$$

For an arbitrary airfoil, both $m$ and $\alpha_{0}$ are determined according to the $C_{L}$ vs. $\alpha$ curve where the constant lift coefficient slope, $m$, is computed over the linear region (attached flow). The modified angle of attack based on the Eq.(12) is used as entry to calculate the lift, the drag and the moment coefficients through the tabulated airfoil data.

As a result, the lift and the drag forces are computed for each blade element in the spanwise section giving the tangential and the normal forces acting on the rotor blade (see figure 7 ).

\section{Results}

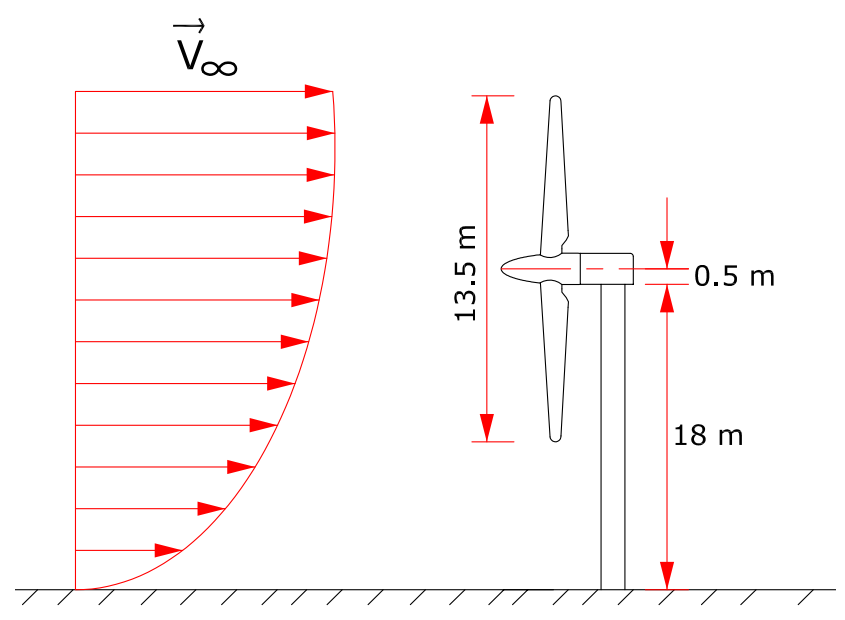

Figure 8. Hönö turbine exposed to upstream flow

The results of the generated power and the thrust vector for the Hönö wind turbine (see table 1) at different yaw conditions are presented. The wind direction is varied between -20 and 20 degrees with a step of 10 degrees, where for each yaw misalignment case, the wind speed is varied from $7 \mathrm{~m} / \mathrm{s}$ to $14 \mathrm{~m} / \mathrm{s}$ with a step of $1 \mathrm{~m} / \mathrm{s}$. The wind shear

\begin{tabular}{|c|c|}
\hline Number of blades & 2 \\
\hline Diameter $[\mathrm{m}]$ & 13.5 \\
\hline Aerodynamic profile & NACA 63-200 \& FFA-W3-xxx \\
\hline Variable rotational speed [rpm] & Max. 75 \\
\hline Rated power $[\mathrm{kW}]$ & 35 \\
\hline Rated wind speed $[\mathrm{m} / \mathrm{s}]$ & 10 \\
\hline
\end{tabular}

Table 1. The Hönö wind turbine specifications

exponent and the angular velocity have been measured during the experiments according to tables 2 and 3 . For all 
simulations done by the VLFW method and the BEM method, it is assumed that the wind turbine is exposed to the vertical shear (see figure 8 ) and the operating conditions is on the basis of tables 2 and 3 . Moreover, the pitch, the tilt and the cone angles are equal to zero for all cases. More information about the blade properties of the Hönö wind turbine can be found in Ref.3.

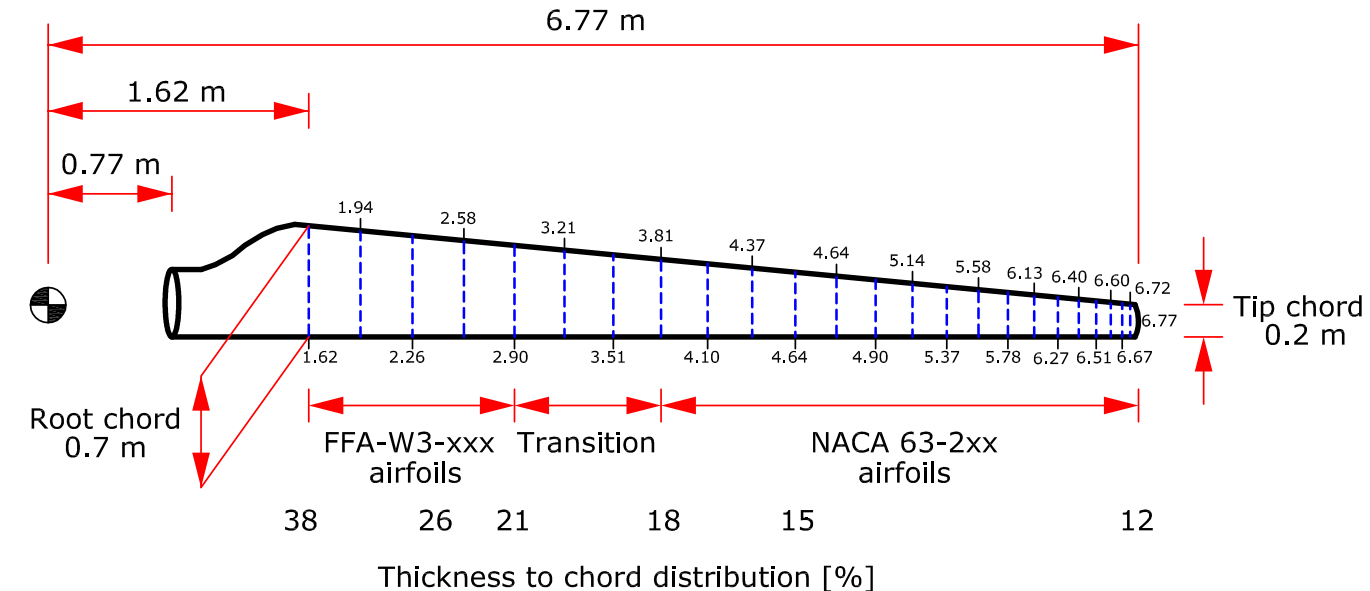

Figure 9. Radial distribution of blade elements

In the vortex method simulations, the blade is discretized with 25 spanwise sections (see figure 9) with fine tip resolution and 11 equally spaced chordwise sections. 10 degrees in the azimuthal direction is employed for the wake segmentation. The wake length is truncated after 4 rotor diameters where the thrust vector is evaluated as the average for the last revolution. It is assumed that the wake vortex filament core radius is constant and is equal to $0.2[\mathrm{~m}]$.

For the BEM method, the blade is discretized with 21 equally spaced spanwise sections. As it was mentioned in the introduction, the skewed wake geometry with trailing vortices ${ }^{7}$ is used to modify the axial induction factor for the yawed flow in the BEM method. ${ }^{2}$ The proposed axial induction correction is applied for each blade element. Since it is a function of blade azimuthal angle, the skewed axial induction factor is taken as the average of one blade revolution with 10 degrees segmentation in the azimuthal direction.

\begin{tabular}{|c|c|c|c|c|c|c|c|c|}
\hline & $7[\mathrm{~m} / \mathrm{s}]$ & $8[\mathrm{~m} / \mathrm{s}]$ & $9[\mathrm{~m} / \mathrm{s}]$ & $10[\mathrm{~m} / \mathrm{s}]$ & $11[\mathrm{~m} / \mathrm{s}]$ & $12[\mathrm{~m} / \mathrm{s}]$ & $13[\mathrm{~m} / \mathrm{s}]$ & $14[\mathrm{~m} / \mathrm{s}]$ \\
\hline$-20[\mathrm{deg}]$ & 7.32 & 7.32 & 7.33 & 7.20 & 7.29 & 7.29 & 7.29 & 7.31 \\
\hline$-10[\mathrm{deg}]$ & 7.32 & 7.33 & 7.28 & 7.27 & 7.22 & 7.25 & 7.29 & 7.30 \\
\hline $0[\mathrm{deg}]$ & 7.16 & 7.29 & 7.25 & 7.17 & 7.09 & 7.14 & 7.21 & 7.33 \\
\hline $10[\mathrm{deg}]$ & 7.27 & 7.30 & 7.29 & 7.24 & 7.14 & 7.16 & 7.08 & 6.86 \\
\hline $20[\mathrm{deg}]$ & 7.18 & 7.29 & 7.32 & 7.34 & 7.21 & 6.88 & 7.08 & 6.86 \\
\hline
\end{tabular}

Table 2. The measured rotational velocity of Hönö wind turbine at different operating conditions

\begin{tabular}{|c|c|c|c|c|c|c|}
\hline Wind speed [m/s] & $4-5$ & $6-7$ & $8-9$ & $10-11$ & $12-13$ & $14-15$ \\
\hline mean wind shear exponent & 0.16 & 0.19 & 0.21 & 0.20 & 0.16 & 0.15 \\
\hline
\end{tabular}

Table 3. The measured mean wind shear exponent at the Hönö wind turbine site

The experimental forces relative to the turbine shaft (see figure 10 ), $\vec{F}_{x}$ and $\vec{F}_{z}$, are calculated on the basis of the bending moments, $M_{x}$ and $M_{t} z$. According to Ref.14, the bending moments $M_{z}$ and $M_{0}$ was measured during two calibration yaw turns where the sideways moment, $M_{z}$, is around zero (assuming no blade rotation) and the nodding moment (due to the blade, hub and nacelle masses called tower top mass) is around the eigenmoment, $M_{0}=$ $-39.5[\mathrm{kNm}]$ both regardless of the yaw position. Since $M_{0}$ is leaning forwards, it must have a negative sign.

The experimental thrust force is computed by

$$
\vec{F}_{z}=\left(M_{x}-M_{0}\right) / L
$$


where $L=16.6[\mathrm{~m}]$ denotes the vertical distance from the center of the hub to the position of the strain gauges. ${ }^{14}$ The experimental transversal force is given by

$$
\vec{F}_{x}=\left(M_{t z}-M_{z}\right) / L
$$

where the value of $M_{z}$ is obtained from the strain gauges attached to the tower. The generator torque $M_{t z}$, is calculated by

$$
M_{t z}=P \cos (\beta) / \Omega
$$

where the tilt angle $(\beta)$ and $P_{\text {sim }}$ denote the angle of the nacelle related to the horizontal plane, which is equal to zero, and the generated power computed by measurement, respectively.

The outcome of the simulations by different methods are the generated power and the thrust due to the aerodynamic forces acting on the rotor blades. In order to calculate the thrust vector angle $(\delta)$ from the simulations, the generated power and the thrust should be translated as the tower bending moments around the $x$ and $z$ axes. Hence, the transversal force from the simulation, $\vec{F}_{x, s i m}$, is given by

$$
\vec{F}_{x, \operatorname{sim}}=\left(\left(P_{\operatorname{sim}} \cos (\beta) / \Omega\right)-M_{z}\right) / L
$$

where $P_{\text {sim }}$ denotes the generated power computed from the simulation. Since the bending moment, $M_{z}$, is related to the tower moment, it is not possible to calculate it from the aerodynamic calculations. Therefore, for comparison between the measurement and the simulation, $M_{z}$ should not be considered in the transversal force equation. Equation (14) is instead modified for both the measurement and the simulation as

$$
\begin{gathered}
\vec{F}_{x}=\left(M_{t z}\right) / L \\
\vec{F}_{x, \text { sim }}=\left(\left(P_{\text {sim }} \cos (\beta) / \Omega\right)\right) / L \\
\vec{F}_{z, \text { sim }}=\left(T_{\text {sim }}-\left(M_{0}\right) / L\right.
\end{gathered}
$$

where $T_{s i m}$ denotes the thrust force calculated from the simulation. As it was mentioned earlier, $M_{0}$ is equal to $-39.5[\mathrm{kNm}]$ provided by the measurements.

The schematic of the resultant thrust vector, $\vec{F}_{R}$, and the thrust deviation angle, $\delta$, are shown in figure 10 .

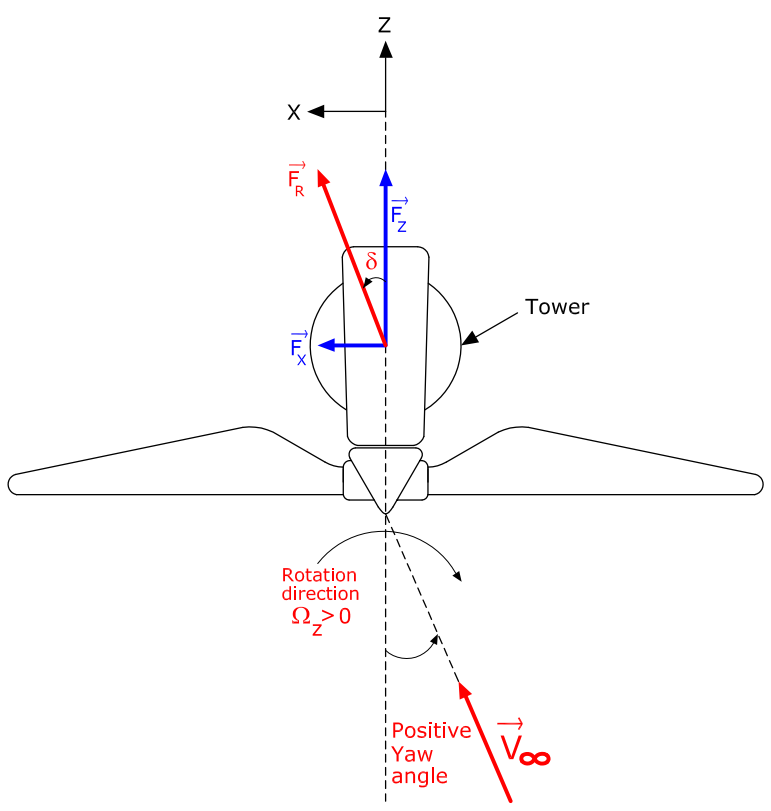

Figure 10. Definition of yawed flow and the thrust vector deviation angle, $\delta$

The experimental data, figure $11 \mathrm{a}$, show that for positive yaw angles and wind speeds higher than $10 \mathrm{~m} / \mathrm{s}$, the thrust vector deviation $(\delta)$ from the shaft axis tends to independent of yaw. For all cases, the thrust vector angle, regarding the loads acting on the rotor blade, is for negative yaw larger than for positive yaw. The generated power measured by 
experiments, figure $11 \mathrm{~b}$, shows the same behavior as the thrust vector deviation $(\delta)$ for wind speed above $10 \mathrm{~m} / \mathrm{s}$. One of the reasons is because of stall occurs for higher wind velocities. In addition, the power reduction due to the yaw misalignment is obvious.
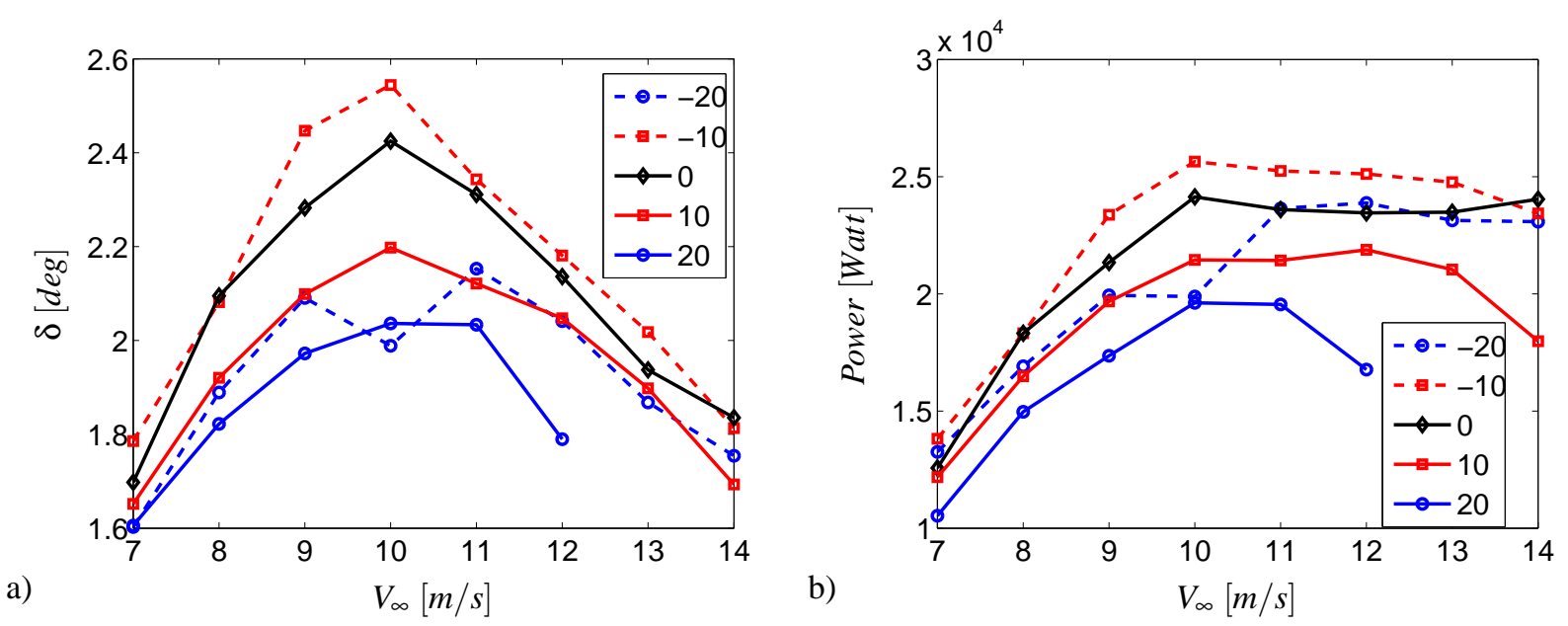

Figure 11. Thrust vector angle and generated power vs. free stream speed for different yaw angles by experiment

Figure $12 a$ shows the variation of the thrust angle based on the standard potential solution of the VLFW method. Unlike for wind speed below $11 \mathrm{~m} / \mathrm{s}$ where $\delta$ for the negative yaw directions is greater than for the positive yaw directions, for the wind speed higher than $11 \mathrm{~m} / \mathrm{s}, \delta$ for the negative yawed flow is smaller than for the positive yawed flow. For most wind speeds, the generated power by the potential solution of the VLFW method for the negative yaw direction is larger than for the positive yaw direction which is expected due to the direction of the rotation (clockwise direction). Moreover, for the zero-yaw condition, the generated power is the largest. In the absence of the viscous drag force for the standard potential method of VLFW, there is no mechanism to reduce the power output due to the stall condition; hence the higher the wind speed, the higher the power (see figure $12 b$ ).
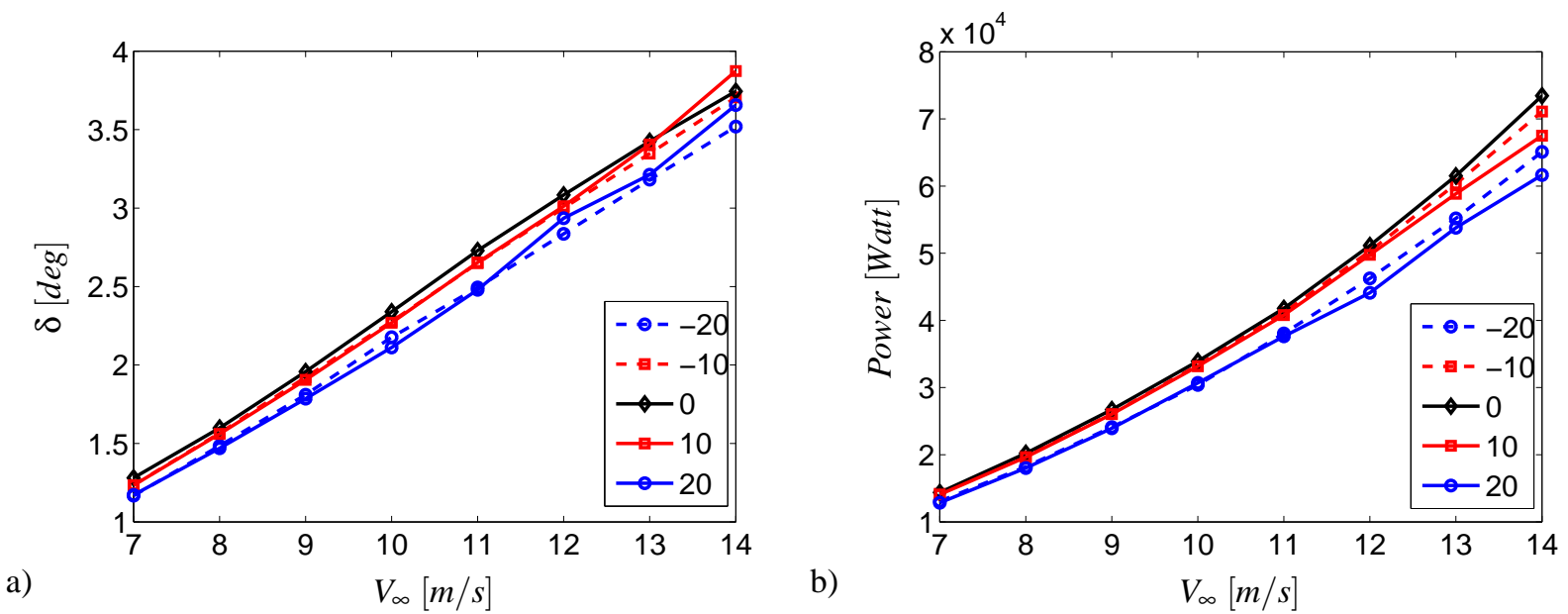

Figure 12. Thrust vector angle and generated power vs. free stream speed for different yaw angles by free wake method (the standard potential method)

According to figure $13 a$, the thrust angle $(\delta)$, based on the $2 D$ static airfoil data method, for the zero-yaw case is the largest while the positive yaw misalignment makes the larger thrust angle than the negative yaw misalignment. Similar to the measurement results, it is seen that $\delta$ tends to be independent of yaw at high wind speeds. There is a good agreement for the generated power between the measurements and the $2 D$ static airfoil data method where the potential solution of the VLFW method is modified by the tabulated airfoil data for each blade element along the blade. For wind speed lower than $11 \mathrm{~m} / \mathrm{s}$, the positive yaw direction of each wind speed generates more power than the negative yaw direction whereas for the wind speed above $11 \mathrm{~m} / \mathrm{s}$, it is inversed. In addition, the generated power for the 10 degree yaw misalignment, both positive and negative directions, is greater than the zero-yaw case for wind speeds higher than $11 \mathrm{~m} / \mathrm{s}$. This behavior is supported by the measurements for negative yaw directions.

Figure 14 presents the results of the BEM method. As can be seen, there is no significant difference for the thrust 

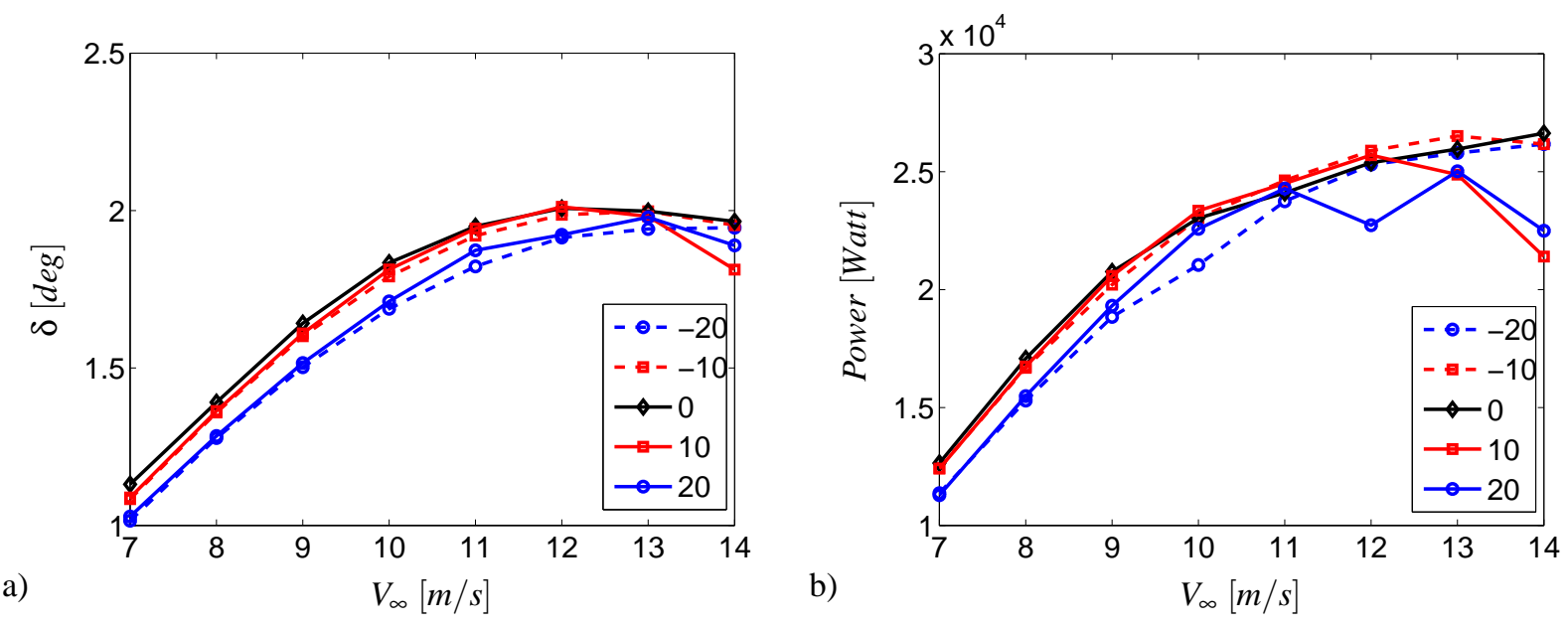

Figure 13. Thrust vector angle and generated power vs. free stream speed for different yaw angles by free wake method (the 2D static airfoil data method)

angle deviation between the different cases. The BEM method also predicts more generated power compared to the measurements and $2 D$ static airfoil data method of VLFW.
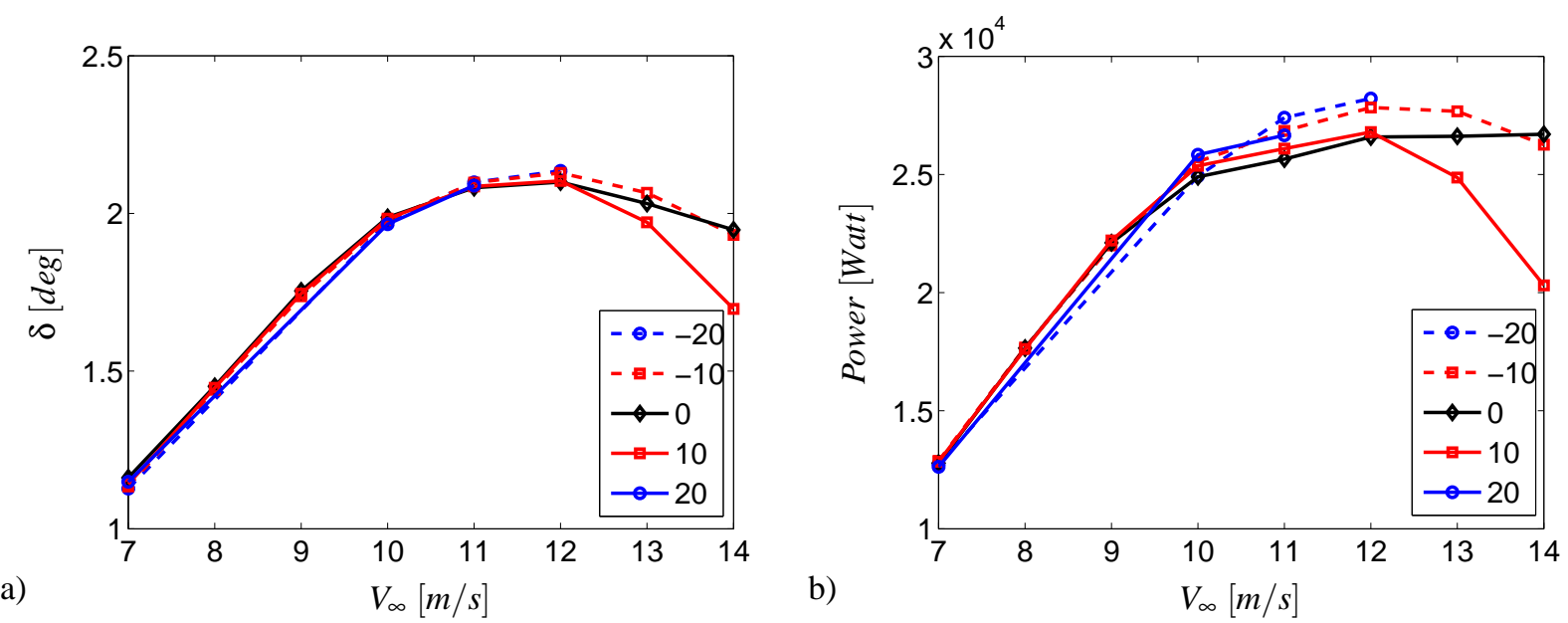

Figure 14. Thrust vector angle and generated power vs. free stream speed for different yaw angles by BEM method

\section{Conclusion}

In the free wake method based on the potential flow, the power is obtained by projection of the lift force, calculated by the Kutta-Jukowsky theory $L^{\prime}=\rho \mathbf{V} \times \Gamma$, on the tangential direction with respect to the rotor plane. The viscous drag force is not taken into account, therefore the predicted power by the standard potential method of VLFW is higher than the other methods. This means that the higher the velocity, the higher the lift force (see figure 12).

Since the simulations have been done on the basis of vertical wind shear, the velocity vectors for all blade elements above the hub height are greater than those below the hub height which results in the load imbalance over the turbine which is generally considered as the source of the yaw moment. Consequently, the angle of attack and the aerodynamic loads at the top of the rotor is greater than at the bottom of the rotor which makes a cyclic variation with period of $1 P$ in the angle of attack.

The rotation direction of the rotor blades plays a major role for the different generated power and thrust angle deviation because of the positive and negative yawed flows. This difference comes from the inplane velocity component of the upstream flow due to the yaw misalignment. This velocity component is both added and subtracted, with respect to the rotation direction, to the rotational velocity of all blade elements. For a wind turbine that rotates in the clockwise direction (viewed from upstream along the rotation axis) such as the Hönö wind turbine, the inplane velocity component, due to the positive yaw condition, is added to the rotational velocity of each blade element above 
the hub height and is subtracted from the rotational velocity of all blade elements below the hub height.

The independency of the BEM method with respect to the yaw misalignment implies that the yaw correction model for the BEM method is insensitive to the turbine rotation direction by averaging over one blade revolution.

The cyclic variation of the angle of attack is considered as one of the unsteadiness sources of wind turbine operating condition. Since the variation in frequency of these sources may be high, the quasi-static aerodynamic is no longer valid. ${ }^{15,16}$ As a future outlook, a dynamic approach must be introduced to modify the aerodynamic coefficients for unsteady operating conditions. This approach which is called Dynamic Stall, adjusts the aerodynamic coefficients for each blade element on the basis of the 2D static airfoil data together with the correction for the separated flow.

\section{Acknowledgments}

The Swedish Wind Power Technology Centre (SWPTC) is a research centre for design of wind turbines. The purpose of the Centre is to support Swedish industry with knowledge of design techniques as well as maintenance in the field of wind power. The research in the Centre is carried out in six theme groups that represent design and operation of wind turbines; Power and Control Systems, Turbine and Wind loads, Mechanical Power Transmission and System Optimisation, Structure and Foundation, Maintenance and Reliability as well as Cold Climate. This project is part of Theme group 2.

SWPTCs work is funded by the Swedish Energy Agency, by three academic and thirteen industrial partners. The Region Västra Götaland also contributes to the Centre through several collaboration projects.

\section{References}

${ }^{1}$ Snel, H. and Schepers, J., Joint Investigation of Dynamic Inflow Effects and Implementation of an Engineering Method, ECN-C-94-107, Petten, Energy Research Centre of the Netherlands April, 1995.

${ }^{2}$ Hansen, M. O., Aerodynamics Of Wind Turbines, 2nd edition, EarthScan, 2008.

${ }^{3}$ Ganander, H. and et al., Variable speed stall regulated HAWT study Hönö 2 Wind Turbine Project, Phase 1, Chalmers University of Technology, Gothenburg, Sweden and Garrad Hassan Ltd, 1991.

${ }^{4}$ van Garrel, A., Development Of A Wind Turbine Aerodynamics Simulation Module, ECN report, ECN-C-03-079, August 2003.

${ }^{5}$ Vermeer, L., Sørensen, J., and Crespo, A., "Wind Turbine Wake Aerodynamics," Progress in Aerospace Sciences, Vol. 39, 2003, pp. 467-510.

${ }^{6}$ Glauert, H., The Analysis of Experimental Results in the Windmill Brake and Vortex Ring States of an Airscrew, RCR R\&M No. 1026, 1926.

${ }^{7}$ Moriarty, P. and Hansen, A., AeroDyn Theory Manual, National Renewable Energy Laboratory, NREL/TP-500-36881, 2005.

${ }^{8}$ Gupta, S., Development Of A Time-Accurate Viscous Lagrangian Vortex Wake Model For Wind Turbine Applications, University of Maryland, Department of Aerospace Engineering, 2006.

${ }^{9}$ Pesmajoglou, S. and Graham, J., "Prediction Of Aerodynamic Forces On Horizontal Axis Wind Turbines In Free Yaw And Turbulence," Journal of Wind Engineering and Industrial Aerodynamics, Vol. 86, 2000, pp. 1-14.

${ }^{10}$ Voutsinas, S., "Vortex Methods In Aeronautics: How To Make Things Work," International Journal of Computational Fluid Dynamics, Vol. 20, 2006, pp. 3-18.

${ }^{11}$ Chattot, J., "Helicoidal Vortex Model For Wind Turbine Aeroelastic Simulation," Computers and Structures, Vol. 85, 2007, pp. $1072-1079$.

${ }^{12}$ Chattot, J., "Optimization Of Wind Turbines Using Helicoidal Vortex Model," Journal of Solar Energy Engineering, Vol. 125, 2003, pp. 418424.

${ }^{13}$ Katz, J. and Plotkin, A., Low-Speed Aerodynamics, Cambridge University Press, 2nd ed., 2001.

${ }^{14} \mathrm{Haag}, \mathrm{C} .$, Wickström, A., and Goldberg, M., Measurements of Hn turbine properties and thrust vector at yaw misalignment, SWE-0023-C, Scandinavian Wind AB, 2013

${ }^{15}$ Leishman, J., "Challenges In Modeling The Unsteady Aerodynamics Of Wind Turbines," 21st ASME Wind Energy Symposium and the 40th AIAA Aerospace Sciences Meeting, Reno, NV, Vol. 0037, 2002.

${ }^{16}$ Bierbooms, W., "A Comparison Between Unsteady Aerodynamic Models," Journal of Wind Engineering and Industrial Aerodynamics, Vol. 39, 1992, pp. 23-33. 